I. Stamatellos $\cdot$ C. Karydas $\cdot$ E. Anagnostou •

P. Stamatopoulos $\cdot$ A. Apostolidis $\cdot$ I. Bontis

\title{
Laparoscopic treatment of premenopausal patients with benign adnexal pathology
}

Received: 3 May 2005 / Accepted: 29 November 2005 / Published online: 14 February 2006

(C) Springer-Verlag Berlin / Heidelberg 2006

\begin{abstract}
The aim of this study was to evaluate the laparoscopic approach to benign adnexal masses in 211 premenopausal patients selected with strict preoperative clinical and ultrasound diagnosed criteria. Most patients were successfully managed with operative laparoscopy $(98.2 \%)$. Four patients underwent laparotomy $(1.8 \%)$. The rate of unexpected adnexal malignancy was $0.48 \%$. We believe that operative laparoscopy is a safe and effective procedure for treating most benign adnexal masses in premenopausal women. To reduce the possibility of encountering an unexpected malignancy when approaching an adnexal cyst by laparoscopy, appropriate preoperative selection of patient candidates for laparoscopic treatment is mandatory. If an unexpected malignancy arises during laparoscopy, a team with specific oncologic training should be available for a possible immediate staging laparotomy in order not to affect the patient's prognosis.
\end{abstract}

Keywords Laparoscopy · Adnexal mass · Ovarian · Cyst · Ovarian malignancy

\section{Introduction}

Laparoscopy is progressively replacing laparotomy for managing most benign gynecological pathology. Com-

I. Stamatellos $(\bowtie) \cdot$ E. Anagnostou

P. Stamatopoulos · A. Apostolidis · I. Bontis

First Academic Department of Obstetrics and Gynaecology, Papageorgiou General Hospital,

Priferiaki Odos, Nea Eukarpia,

Thessaloniki, 54606, Greece

e-mail:stamio@mail.com

I. Stamatellos · C. Karydas · E. Anagnostou - A. Apostolidis Geniki Kliniki,

Thessaloniki, 2 Grabias-54645, Greece

I. Stamatellos · C. Karydas · E. Anagnostou - A. Apostolidis Interbalkan Medical Centre,

Thessaloniki, 57001, Greece pared with laparotomy, laparoscopy has more benefits for the patient and reduces health care costs. Patients benefit from a shorter recovery time, reduced need for analgesia, and less cosmetic deformity. The cost of health service is reduced because of shorter hospital stays. Many of the laparoscopic operations are carried out as day-case procedures, and costs can be further reduced by using a dedicated day-surgery unit in the hospital.

Because of increased surgical expertise and technological evolution, the laparoscopic approach has become an established practice for managing benign adnexal masses. However, surgeons face the possibility of encountering an unexpected ovarian malignancy at the time of operation [1]. If the ovarian malignancy is diagnosed at the beginning of the diagnostic laparoscopy, an immediate staging laparotomy should be performed, with no detrimental effect on the patient's prognosis. Two of the main concerns regarding laparoscopic assessment of ovarian masses are the possibility of missing a malignancy during operative laparoscopy and the consequences of malignant cyst rupture (or malignant cyst biopsy) on the patient's prognosis. If the ovarian malignancy is recognized during laparoscopy after the cyst has already ruptured, concerns have been reported regarding the patient's prognosis, even if an immediate laparotomy follows the laparoscopy. Evidence suggests that cyst rupture at the time of surgery does not influence the prognosis for malignant neoplasms, provided that definitive surgery is carried out without delay [2].

For these reasons, appropriate selection of patients is critical for diminishing the possibility of encountering an unexpected malignancy during laparoscopic management of adnexal masses. In this study, we report results concerning 211 patients with a diagnosis of benign ovarian cyst who had laparoscopic treatment in the last 4 years.

\section{Materials and methods}

Between February 2000 and December 2004, 211 patients aged 17-42 years with ultrasound and clinical findings of 
benign adnexal cyst were selected by the authors for laparoscopic management. The diagnoses were made during gynecological examinations and diagnostic workups for menstrual irregularities, secondary dysmenorrhea, infertility, and acute and chronic pelvic pain. All patients were premenopausal.

The investigation of an adnexal cyst requires differentiating it as benign or malignant, something that is not always possible. Our study's inclusion criteria for laparoscopic management were ultrasound features suggestive of malignancy, as shown in Table 1. Biochemical serum markers including CA-125 were elevated in 167 cases, with a range of $38-81 \mathrm{ml} / \mathrm{l}$, but it is well established that elevated CA-125 does not necessarily imply cancer, and the marker may be normal in the presence of ovarian epithelial malignant tumor [3]. Serum CA-125 is more reliable in postmenopausal patients when combined with pelvic ultrasound [4-6] because in premenopausal patients, it may be elevated in a range of benign conditions (endometriosis, pelvic inflammatory disease, adenomyosis, benign ovarian cysts). For this reason, CA-125 values were recorded but not used in the set of inclusion criteria.

All procedures were performed under general anesthesia. After pneumoperitoneum was established, an 11-mm umbilical trocar was inserted and a 10-mm laparoscope introduced. Before the laparoscopic management of the ovarian cyst, peritoneal washings for cytology were taken, the upper abdomen and pelvis were carefully inspected, and the procedure was converted to a staging laparotomy if a malignancy was suspected or diagnosed. Intraoperatively, removal of the cyst without spillage was attempted, efficient peritoneal lavage at the end of surgery was done, and biopsies were taken of any suspicious areas and the collected samples sent for frozen section analysis.

After laparoscopic inspection that suggested a benign nature of the adnexal mass, the cyst capsule was separated in most cases, and an intact cystectomy was performed. Using a combination of sharp, blunt, and aqua dissection, a cleavage plan was found until the cyst was shelled out intact. The ovary was left unsutured, and bleeding points were coagulated with bipolar diathermy. When no sign of

Table 1 Transvaginal ultrasound findings indicative of malignancy

Ultrasound finding

Size of mass $>10 \mathrm{~cm}$

Bilaterality

Thick capsule

Thick septa $(>3 \mathrm{~mm})$

No smooth outline of cyst

Solid components

Papillary excrescences on ovary

Ascites or hepatomegaly

Metastases

Doppler signal showing reduced pulsatility index ${ }^{\mathrm{a}}$

${ }^{a}$ Color-flow Doppler was used to identify neovascularization inside ovarian cysts. This was observed in only one patient involved in this study possible malignancy was observed, the cyst was punctured with a 5-mm trocar and cannula. After the trocar was withdrawn, the suction instrument was inserted down the cannula, remaining inside the cyst. The contents were then aspirated and the cyst removed, using the laparoscopic grasping forceps through one of the lateral ports after the trocar sleeve removal. Alternatively, upon completion of the cystectomy, the cyst could be removed from the abdomen with an endoscopic bag (LapSac, Cook Urological, and Endobag, Tyco Healthcare), the bag being drawn through the lateral port incision under laparoscopic control.

If the cyst was ruptured during the procedure, an open cystectomy was performed. This approach was often used in endometriotic cysts. At the end of the procedure, copious irrigation with warmed Ringer's solution or normal saline, with the patient in both Trendelenburg and reverse Trendelenburg position, was done to remove all the debris from the subdiaphragmatic area in the upper abdomen and the pelvic cavity.

During open cystectomy, the cyst wall was removed using two grasping forceps working closely together and stripping the cyst wall off the ovary.

After the cyst was removed, the specimen was inspected outside the pelvis for signs of malignancy. If any were noted, the operation was converted to a staging laparotomy. An experienced histopathologist was available for accurate frozen section analysis. Close cooperation with an oncologist was also available on-site for optimization of the patient's outcome.

\section{Results}

The mean age of the 211 patients was 29.2 years (range 1742 years), and the mean diameter of the ovarian cysts was $6.1 \mathrm{~cm}$ (range 3.4-9.7 cm). The histologic results are shown in Table 2. During diagnostic laparoscopy, three patients were diagnosed with stage 4 endometriosis and frozen pelvis. The operations in these cases were completed by laparotomy. In one patient with a large dermoid cyst ( $8.2 \mathrm{~cm}$ in diameter) with a very thick base, a minilaparotomy was performed (extending the incision of

Table 2 Histologic results from the 211 premenopausal patients treated by laparoscopy

\begin{tabular}{lcl}
\hline & Number of cysts & Percentage \\
\hline Serous cyst & 72 & $(34.13 \%)$ \\
Endometriotic cyst & 68 & $(32.23 \%)$ \\
Paraovarian cyst & 7 & $(3.32 \%)$ \\
Hemorrhagic cyst & 11 & $(5.22 \%)$ \\
Cystadenomas & 9 & $(4.27 \%)$ \\
Dermoid cyst & 14 & $(6.64 \%)$ \\
Hydrosalpinx & 6 & $(2.90 \%)$ \\
Primary tubal carcinoma & 1 & $(0.48 \%)$ \\
Follicular cyst & 19 & $(9.10 \%)$ \\
Lutein cyst & 4 & $(1.90 \%)$ \\
\hline
\end{tabular}


the suprapubic portal) to remove the tissue and also to avoid extensive spillage of the contents.

One patient, 42 years old with a history of breast carcinoma and 8 months of tamoxifen therapy after modified radical mastectomy, presented with menstrual problems. During the ultrasound examination to assess endometrial thickness, a cyst in her left ovary was incidentally revealed, with a small papillomatous area $(0.8 \mathrm{~cm})$ and increased vascularization of this point. There were no other ultrasound or clinical signs suggestive of malignancy. Diagnostic laparoscopy revealed a left hydrosalpinx. A left salpingectomy was performed, and the specimen was removed from the abdomen using the "in a bag" technique. The frozen section analysis and the histologic result was primary tubal adenocarcinoma, a rare malignancy accounting for $0.18-1.8 \%$ of all gynecological malignancies $[7,8]$. After that, a total abdominal hysterectomy with bilateral oophorectomy and right salpingectomy was performed, followed by omentectomy. Finally, diaphragmatic scrapes were taken, and selective pelvic and paraaortic lymphadenectomy was performed.

Intact cystectomy was performed in 38 patients $(18 \%)$. Open cystectomy was performed in 166 patients (78.6\%). Removal of the cyst using the "in a bag" technique was done in 56 patients $(26.5 \%)$.

Laparoscopic unilateral oophorectomy was done in two patients with multiple endometriotic cysts that had destroyed almost all the ovarian parenchyma. After operative laparoscopy for endometriotic cysts, these patients were treated with 3 months of GnRH analog therapy.

Intraoperative complications included two cases of trauma of the inferior epigastric vessels during the insertion of the lateral trocars. These cases were managed successfully with bipolar diathermy during the operation and suturing under laparoscopic control according to Chatzipapas's technique [9].

One patient with adhesions between the ovary and bowel had rupture of the serosa of the sigmoid during dissection. Conservative management was successful.

Hospital stays ranged from 15 to $48 \mathrm{~h}$ (mean 22.6 hours). Most patients (95.3\%) were discharged within $24 \mathrm{~h}$, and $2.9 \%$ were discharged within $48 \mathrm{~h}$. The four patients who had a laparotomy (three with advanced endometriosis and one with primary tubal carcinoma) were discharged within 4 days $(1.8 \%)$.

Postoperative complications included an umbilical incision inflammation after 1 week from the laparoscopic treatment for a dermoid cyst excision. Conservative management with antibiotics and local antiseptic application proved to be efficient.

The follow-up periods ranged from 6 to 48 months.

Cyst recurrence occurred after 6 months in four endometriotic cysts $(1.8 \%)$, and these patients were treated with second-look laparoscopy and open cystectomy (cystotomy and stripping of the cyst wall).

\section{Discussion}

The results of this study suggest that laparoscopic treatment of an ovarian mass can be performed successfully in most patients if strict patient selection criteria are followed. A timely conversion to staging laparotomy when malignant disease is suspected is necessary to ensure that the prognosis is not compromised in the case of ovarian cancer.

Ultrasound scanning [10], particularly using a vaginal probe, facilitates detailed study of ovarian cysts. The use of Doppler imaging may increase the sensitivity of this approach. Mainman et al. [1] reviewed 42 cases of ovarian cancer that were suboptimally managed laparoscopically, although preoperative investigations had been studied and ultrasound features of possible malignancy had been considered inclusive. Thirty-one percent of the malignant cysts in the series showed benign characteristics (size $<8 \mathrm{~cm}$, cystic nature, unilocular and unilateral presentation). These findings suggest that ultrasound cannot reliably discriminate between benign and malignant ovarian cysts and that the surgeon should manage these cases according to specific protocol.

However, a simple cyst in a woman younger than 35 years of age has a 4.5 in 100,000 chance of being malignant [11]. The incidence of ovarian malignancy in women less than 40 years is very low (within a range of $0.8-8.6$ per 100,000 women per year), but this incidence increases with patient age [12].

Several studies have shown that is possible to assess suspicious adnexal cysts during laparoscopy. Nezhat et al. [13] (1,011 cases), Mettler et al. [14] (626 cases), Mage et al. [15] (481 cases), and Canis et al. [16] (757 cases) reported that no ovarian cancers were missed during operative laparoscopy for ovarian cysts.

Highly suspicious adnexal cysts can be evaluated laparoscopically. The chance of proceeding to laparotomy is increased. That was the case of one patient in this study with a final diagnosis of primary tubal adenocarcinoma. Immediate staging laparotomy performed without delay does not affect the patient's prognosis $[2,17]$.

However, a cyst that demonstrates some features suggestive of malignancy may be benign. Statistically, the larger the cyst, the greater the possibility of malignancy; also, ovarian cysts larger than $5 \mathrm{~cm}$ have been linked with a higher risk of malignancy [18-20], but it is unknown which size discriminates most accurately between benign and malignant cysts. Some authors' expressed views that laparoscopy is inappropriate for women with solid, multiloculated, or large adnexal masses may increase the possibility of these women undergoing laparotomy even for benign diseases. These ultrasound-diagnosed features can be seen in dermoid cysts or endometriomas. In contrast, some ovarian cancers show benign characteristics on preoperative assessment. 
For these reasons, all cysts should be treated as suspicious because this will lead to a consistent laparoscopic approach. Internal papillae and neovascularization are features more suggestive of malignancy, as reported in this study. The rate of unexpected malignancies in this study was $0.48 \%$, similar to the rates of larger series, which report a range of $0.2-2.5 \%$ [21].

Cyst rupture percentage was $78.6 \%$ in this study, but this is a frequent phenomenon during laparoscopy. This event is also common during laparotomy performed by experienced surgeons with similar rupture rates for laparoscopy and laparotomy [22] or lower rupture rates for laparotomy $[23,24]$.

The cyst recurrence rate in this study at 6 months was $1.8 \%$, which is consistent with other reported values [25]. All of these cysts were endometriotic.

In conclusion, laparoscopic treatment of benign adnexal masses in premenopausal women is a safe and effective procedure in most cases. The surgeon must keep in mind the possibility of an unexpected ovarian malignancy and perform appropriate intraoperative management. Careful preoperative evaluation of patients should identify women who have suspicious adnexal masses and, therefore, an increased likelihood of needing an immediate staging laparotomy.

\section{References}

1. Maiman M, Seltzer V, Boyce J (1991) Laparoscopic excision of ovarian neoplasms subsequently found to be malignant. Obstet Gynecol 77:563-565

2. Dembo A, Davy M, Stenwing A et al (1990) Prognostic factors in patient with stage I epithelial ovarian cancer. Obstet Gynecol 75:263-272

3. Wong YM, Amer S, Li TC et al (2000) Laparoscopic management of ovarian cysts. Gynaecol Endosc 9:79-90

4. Einhorn N, Bast RC, Knapp RC et al (1986) Preoperative evaluation of serum CA 125 levels in patients with primary epithelial ovarian cancer. Obstet Gynecol67:414-416

5. Malkasian GD, Knapp RC, Lavin PT et al (1988) Preoperative evaluation of serum CA 125 levels in premenopausal and postmenopausal patients with pelvic masses: discrimination of benign from malignant disease. Am J Obstet Gynecol 159: 341-346

6. Patsner B, Mann WJ (1988) The value of preoperative serum CA 125 levels in patients with a pelvic mass. Am J Obstet Gynecol 159:873-876

7. Hu CY, Taymor ML, Hertig AT (1950) Primary carcinoma of the fallopian tube. Am J Obstet Gynecol 58:58-67

8. Mc Murray EH, Jacobs AJ, Perez CA et al (1986) Carcinoma of the fallopian tube. Management and sites of failure. Cancer 58:2070-2075
9. Chatzipapas IK, Magos AL (1997) A simple technique of securing inferior epigastric vessels and repairing the rectus sheath at laparoscopic surgery. Obstet Gynecol 90(2):304-306

10. Timer-Trisch I, Lerner J, Santos R (1993) Transvaginal ultrasonography characterisation of ovarian masses by means of colour flow-directed Doppler measurements and a morphologic scoring system. Am J Obstet Gynecol 168:909-913

11. United States Department of Health and Human Services (1984) Cancer incidence and mortality in the United States. SEER, 1973-81. NIH publication no. 85-1837. Public Health Service, National Institutes of Health, Bethesda, pp 55, 207

12. Goodman MT, Howe HL (2003) Descriptive epidemiology of ovarian cancer in the United States, 1992-1997. Cancer 97 (S):2615-2630

13. Nezhat F, Nezhat C, Silfen S et al (1992) Four ovarian cancers diagnosed during laparoscopic management of 1011 women with adnexal masses. Am J Obstet Gynecol 167:790-796

14. Mettler L, Caesar G, Neunzling S et al (1993) Value of endoscopic ovarian surgery-critical analysis of 626 pelviscopically operated ovarian at the Kiel University Gynecologic Clinic 1990-1991 [German]. Geburtshilfe Frauenheilk 53: 253-257

15. Mage G, Wattiez A, Canis M et al (1991) Contribution of celioscopy in the early diagnosis of ovarian cancers [French]. Ann Chir 45:525-528

16. Canis M, Mage G, Pouly JL et al (1994) Laparoscopic diagnosis of adnexal cystic masses: a 12-year experience with long term follow-up. Obstet Gynecol 83:707-712

17. Lehner R, Wenzl R, Heinzl H et al (1998) Influence of delayed staging laparotomy after laparoscopic removal of ovarian masses later found to be malignant. Obstet Gynecol 92: 967-971

18. Sassone AM, Timor-Tritsch IE, Artner A et al (1991) Transvaginal sonographic characterisation of ovarian disease: evaluation of a new scoring system to predict ovarian malignancy. Obstet Gynecol 78:70-76

19. Rulin MC, Preston AL (1987) Adnexal masses in postmenopausal women. Obstet Gynecol 70:578-581

20. Granberg S, Wikland M, Janson I (1989) Macroscopic characterization of ovarian tumours and the relation to the histologic diagnosis: criteria to be used for ultrasound evaluation. Gynecol Oncol 35:139-144

21. Muzii L, Angioli R, Zullo M et al (2005) The unexpected ovarian malignancy found during operative laparoscopy: incidence, management, and implications for prognosis. J Minim Invasive Gynecol 12(1):80-89

22. Yuen PM, Yu KM, Yip S et al (1997) A randomized prospective study of laparoscopy and laparotomy in the management of benign ovarian masses. Am J Obstet Gynecol 177:109-114

23. Lin P, Falcone T, Tulandi T (1995) Excision of ovarian dermoid cyst by laparoscopy and by laparotomy. Am J Obstet Gynecol 173:769-771

24. Gal D, Lind L, Lovecchio JL et al (1995) Comparative study of laparoscopy vs. laparotomy for adnexal surgery: efficacy, safety, and cyst rupture. J Gynecol Surg 11:153-158

25. Marana R, Muzii L, Catalano GF et al (2004) Laparoscopic excision of adnexal masses. J Am Assoc Gynecol Laparosc 11 (2):162-166 